\title{
Microcompósitos de poliuretano contendo resíduo wet blue da indústria coureira, por polimerização in situ
}

\author{
Dandara Dessimon ${ }^{\prime}$ \\ Leonardo Chiká \\ Leonardo Moreira dos Santos ${ }^{2}$ \\ Sandra Einloft ${ }^{2}$ \\ Fernando Morisso ${ }^{1}$ \\ Claudia Trindade Oliveira ${ }^{l}$ \\ Leonardo Felix Scheffels ${ }^{1}$ \\ Sandra Raquel Kunst ${ }^{3 *}$ \\ Patrice Monteiro de Aquim ${ }^{1}$ \\ Carlos Leonardo Pandolfo Carone ${ }^{l}$ (1)
}

\section{Resumo}

O presente trabalho refere-se ao estudo da incorporação de um resíduo sólido gerado pela indústria coureira, chamado de serragem de couro wet blue, oriundo da operação de rebaixe que uniformiza a espessura do mesmo, a uma matriz de poliuretano, formando microcompósitos. Este resíduo é classificado como resíduo Classe I pela norma ABNT NBR 10.004, de 2004. Portanto, o objetivo desta pesquisa é o reaproveitamento deste resíduo, incorporando-o, como reforço, em matriz de poliuretano. O método consiste na moagem e no peneiramento da serragem de couro, que passa pelo processo de secagem em estufa, para ser adicionado durante o processo de polimerização in situ. Esta carga foi incorporada nas proporções de $0,5,2,0$ e 5,0\% em massa do polímero puro. Com isso, foi verificada a modificação nas propriedades térmicas do compósito formado, por meio das análises de FT-IR, TGA, DSC, DRX e MEV. Como resultados, verificou-se o aumento da cristalinidade e, consequentemente, o aumento da resistência térmica conforme aumentou-se a concentração da carga de cada microcompósito. Portanto, esta alternativa mostrou-se uma possibilidade para a reciclagem do resíduo de couro wet blue.

Palavras-chave: Microcompósito; Poliuretano; Polimerização in situ; Wet blue; Resíduo.

\section{Polyurethane microcomposites containing wet blue residue from the leather industry by in situ polymerization}

\begin{abstract}
The present work refers to the study of the incorporation of a solid residue generated by the leather industry, called wet blue leather sawdust originating from the countersink operation which standardizes the thickness thereof, in a polyurethane matrix to form microcomposites. This waste is classified as Class I residue by the standard NBR 10.004, of 2004. Therefore, the objective of this research is the reuse of this waste, incorporating it as reinforcement, in polyurethane matrix. The method consists of grinding and sieving the leatherdust, which must pass through the oven drying process, to be added during the in situ polymerization process. This charge was incorporated in proportions of $0.5,2.0$ and $5.0 \%$ by mass of the pure polymer. Thus, the modification will be verified in the thermal properties of the composite formed, by means of the FT-IR, TGA, DSC, XRD and MEV analyzes. As a result, there was increased crystallinity and, consequently, increased thermal resistance as the concentration of the charge of each microcomposite increases. Therefore, this alternative proved to be a possibility for the destination of wet blue leather residue.
\end{abstract}

Keywords: Microcomposite; Polyurethane; In situ polymerization; Wet blue residue.

${ }^{1}$ Universidade Feevale, Novo Hamburgo, RS, Brasil.

${ }^{2}$ Pontificia Universidade Católica do Rio Grande do Sul-PUCRS, Porto Alegre, RS, Brasil.

${ }^{3}$ Lacor, Universidade Federal do Rio Grande do Sul-UFRGS, Porto Alegre, RS, Brasil.

*Autor correspondente: tessaro.sandra@gmail.com

2176-1523 (C) 2020. Dessimon et al. Publicado pela ABM. Este é um artigo publicado em acesso aberto (Open Access) sob a licença Creative Commons Attribution, que permite uso, distribuição e reprodução em qualquer meio, sem restrições desde que o trabalho original seja Commons Attribution
corretamente citado. 


\section{Introdução}

A indústria coureira é responsável por transformar a pele natural (verde ou salgada) em couro. Dentre eles destaca-se o resíduo sólido de wet-blue, pois são curtidos com cromo. Esses resíduos são classificados como sendo de Classe I de acordo com a norma NBR 10004 (ABNT, 2004) [1], e o seu principal destino são os aterros de resíduos industriais perigosos. De acordo com o Centro Comercial das Indústrias de Curtumes do Brasil [2], o setor calçadista destaca-se nas regiões Sul e Sudeste, sendo responsáveis por aproximadamente $72 \%$ da produção total de couro, além de que, registram o maior número de curtumes no pais. A indústria coureira-calçadista, destaca-se como uma das maiores geradoras de resíduos sólidos de Classe I, do estado do Rio Grande do Sul. De acordo com a FEPAM [3], a indústria de couros e peles é responsável por gerar 128.578 toneladas por ano de resíduos sólidos industriais perigosos, no Rio Grande do Sul. No processo produtivo de 1 tonelada de couro gera aproximadamente $377 \mathrm{~kg}$ de resíduo, sendo que destes $225 \mathrm{~kg}$ correspondem a serragem de couro wet-blue [4]. Salienta-se que este resíduo tem como característica apresentar um alto potencial de impacto ambiental, tendo em vista que, $o$ mesmo no seu processamento é curtido com cromo trivalente $\left(\mathrm{Cr}^{+3}\right)$, no qual pode oxidar-se e formar cromo hexavalente $\left(\mathrm{Cr}^{+}\right)$. Sendo assim, torna-se necessário, que se estabeleçam procedimentos ou métodos de reutilização destes resíduos no sentido de minimizar este impacto ambiental $[5,6]$. Cabe mencionar que o cromo (VI) causa sérios riscos à saúde e é classificado como cancerígeno [6,7]. Além disso, o resíduo é composto por colágeno, cujo comprimento e o diâmetro de uma molécula são de, respectivamente, $280 \mathrm{~nm}$ e 1,4 nm. Uma microfibrila e uma fibrila de colágeno tem diâmetros de $40 \mathrm{~nm}$ e 100 a 200 nm, respectivamente [8].

Têm-se buscado alternativas que sejam viáveis economicamente, como tratamento, reciclagem, reaproveitamento e redução da geração de resíduos. Conforme Bañón et al. [7], existem, atualmente, inúmeras linhas de pesquisa que têm como objetivo a busca por processos de tratamento adequados para os resíduos gerados no processamento de couro. Através do tratamento adequado, é possível minimizar os impactos ambientais e obter coprodutos que podem compensar o custo econômico do processo. Dessa forma, neste trabalho, estudou-se o reaproveitamento da serragem de rebaixamento, na forma de micropartículas, adicionando-as a uma matriz polimérica, com o objetivo de formar um material compósito. O resíduo de couro wet blue como carga de compósitos, pode ser classificado como híbrido orgânico/inorgânico, conforme as descrições de Guo et al. [9] e Essabir et al. [10], devido à presença do colágeno (orgânico) e do óxido de cromo (III) (inorgânico). Para a caracterização e utilização como cargas, o resíduo deve ser moído em moinho de facas [11]. Escolheu-se, como matriz polimérica, o poliuretano que é considerado um dos mais importantes materiais poliméricos na engenharia, principalmente por sua versatilidade de aplicação em muitos setores industriais. Como exemplos, pde-se citar sua utilização na construção civil, na indústria de adesivos, na indústria automotiva, nas embalagens em geral, no armazenamento e no transporte, nos revestimentos, e na indústria têxtil e calçadista [12]. O motivo da ampla e versátil aplicação deste polímero se dá pelas suas propriedades, principalmente, propriedades mecânicas como, a resistência à abrasão e ao rasgo, a alta absorção de choques mecânicos e a boa flexibilidade e elasticidade. Contudo, existem outras que limitam a aplicação deste material, como a alta permeabilidade e a baixa estabilidade térmica. Por este motivo, a adição de cargas, em escala micrométrica ou nanométrica, ao polímero tornou-se uma alternativa para melhorar suas propriedades [13]. Há também a possibilidade das cargas serem híbridas orgânica/inorgânica, cujas propriedades apresentadas são únicas em razão da sinergia entre elas e a matriz do compósito [9]. Em estudo sobre a incorporação de fibra de palmeira e argila em polietileno de alta densidade, Essabir et al. [10] concluíram que o compósito híbrido obtido apresentou dispersão de cargas homogênea, melhoria de $49 \%$ no módulo de Young e de 11\% na resistência à tração. Esta pesquisa vem ao encontro às necessidades do momento atual, em que se tornou imprescindível a compreensão e a otimização das combinações de materiais, que podem ter funções inovadoras ou substituir materiais clássicos em suas funções devido as propriedades mais desejáveis.

Compósitos em escalas micrométricas permitem ainda mais o desenvolvimento tecnológico das combinações de materiais, pois com eles é possível a exploração de novas funções e propriedades diferenciadas.

\section{Materiais e métodos}

O resíduo utilizado no presente trabalho é oriundo do processo do couro, fluxograma apresentado na Figura 1, o mesmo é obtido da operação de rebaixe que tem como função uniformizar o couro.

A preparação do resíduo foi realizada nos laboratórios de Reciclagem e Beneficiamento da Universidade Feevale, por meio das seguintes etapas: secagem (estufa, $40^{\circ} \mathrm{C} / 24$ horas), micronização (moinho de facas/1670 rpm), peneiramento (peneira / mesh 3.5). A determinação do teor de óxido de cromo foi realizada em duplicata, conforme a norma NBR 11.054/99 [14]. A incorporação do resíduo foi realizada por meio de reações de polimerização in situ entre um poliol e um diisocianato. As mesmas foram realizadas no Laboratório de Estudos Avançados em Materiais da Universidade Feevale, O poliol utilizado foi o policaprolactona diol (PCL) (2000 g/mol - Aldrich), hexametileno diisocianato (HDI) (Fluka Analytical), em uma razão molar $\mathrm{NCO} / \mathrm{OH}$ 1:1. O catalisador utilizado foi dibutil dilaurato de estanho (DBTDL) (Aldrich) $(0,1 \%$ relativo ao peso de PCL) e metil etil cetona (MEK) (Dinâmica) usado como solvente $(50 \mathrm{~mL})$. O resíduo que foi adicionado ao reator, nas proporções de 0,5, 2,0 e 5,0\% em massa em relação ao polímero puro. As reações foram realizadas em um reator de vidro $(500 \mathrm{~mL})$ com cinco entradas, em que foram acoplados, um agitador mecânico, um condensador de refluxo, um funil 


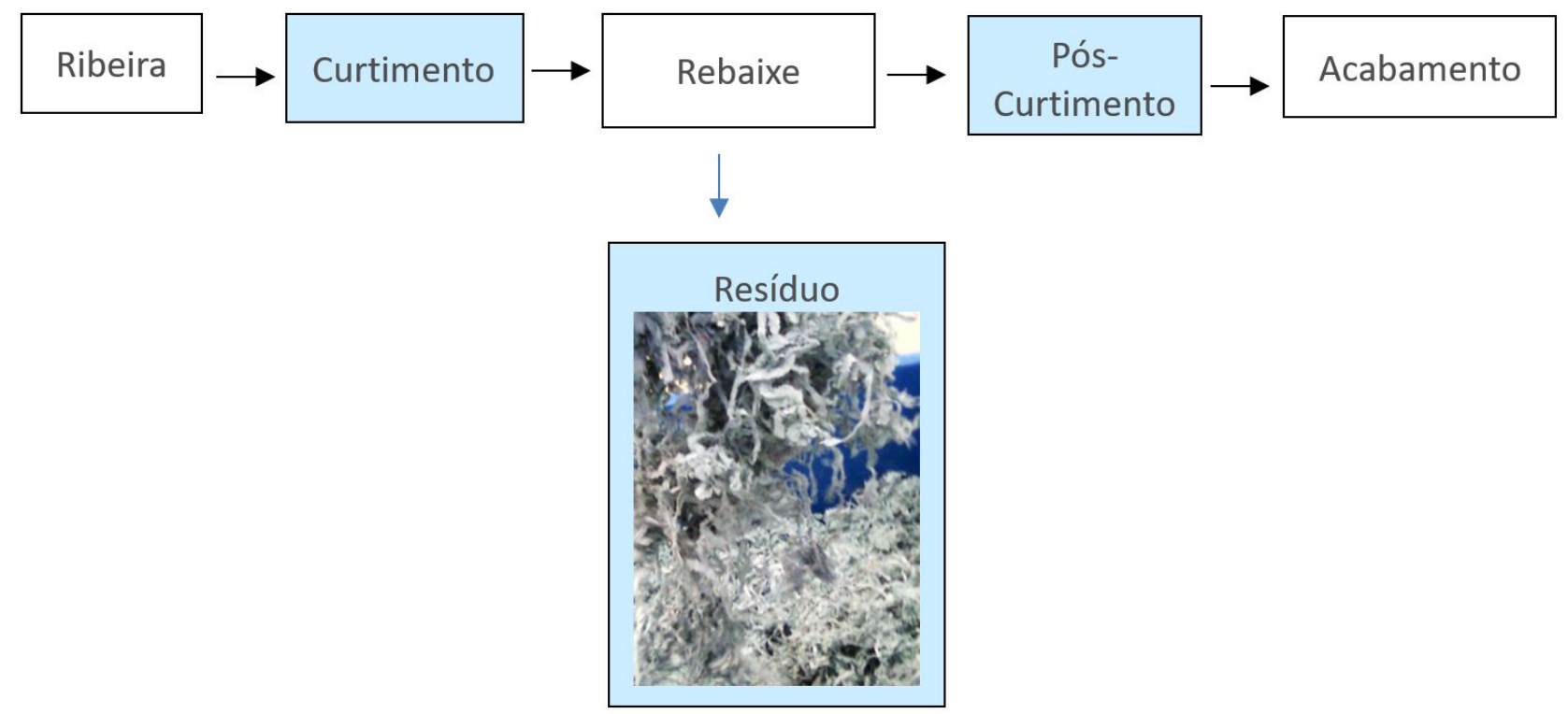

Figura 1. Processo do couro.

de adição e um termopar. Os reagentes foram adicionados no início da reação, sob atmosfera de nitrogênio e a $40{ }^{\circ} \mathrm{C}$. O tempo de reação foi de 1 hora e 30 minutos.

A carga e os microcompósitos foram caracterizados por espectroscopia de infravermelho por transformada de Fourier (FTIR-Perkin Elmer, modelo Spectrum100, usando UATR de 4000 a $650 \mathrm{~cm}^{-1}$ ) onde se avalia a incorporação da carga na matriz polimérica, confirmando a incorporação por comparação dos compósitos com o polímero puro. Análises termogravimétricas foram realizadas em um equipamento da marca Perkin Elmer, modelo STA 6000. A condição utilizada foi de aquecimento de $10{ }^{\circ} \mathrm{C} / \mathrm{min}$ com temperatura variando de $30^{\circ} \mathrm{C}$ a $1000{ }^{\circ} \mathrm{C}$ em atmosfera de nitrogênio com vazão de $20 \mathrm{~mL} / \mathrm{min}$. A temperatura de fusão (Tm) foi avaliada por calorimetria de varredura diferencial (DSC) (equipamento Perkin Elmer modelo DSC 6000). As análises foram realizadas em dois ciclos de aquecimento-resfriamento. A escala de temperatura de -90 a $200^{\circ} \mathrm{C}$ com uma taxa de aquecimento e resfriamento de $10{ }^{\circ} \mathrm{C} / \mathrm{min}^{-1} \mathrm{sob}$ atmosfera de $\mathrm{N}_{2}$. A cristalinidade do polímero puro e dos compósitos formados foi foi analisada por difração de raios X (XRD) usando um difratograma PHILIPS (modelo X'Pert MPD), 40 kV, $40 \mathrm{~mA}$ e anodo de $\mathrm{Cu}$.

As análises de microscopia eletrônica de varredura foram realizadas em um equipamento da marca JEOL, Modelo JSM-6510LV, com tensão de $10 \mathrm{kV}$, em elétrons secundários (SE) e utilizadas para avaliação da distribuição de enchimentos na matriz polimérica. As amostras foram colocadas em uma fita de carbono e cobertas com uma fina camada de ouro.

\section{Resultados e discussões}

Os resultados das análises dos teores de matéria volátil, de cálcio em base úmida e base seca e de óxido de cromo em base úmida e base seca estão apresentados na Tabela 1 .
Tabela 1. Resultados das análises de caracterização do resíduo de couro

\begin{tabular}{cc}
\hline ANÁLISE & RESULTADO \\
\hline Matéria volátil \%(p/p) & 19,47 \\
Cálcio $\%(\mathrm{p} / \mathrm{p})$ & Base úmida 0,122 \\
& Base seca 0,151 \\
Óxido de cromo $\%(\mathrm{p} / \mathrm{p})$ & Base úmida 3,23 $\mathrm{Cr}_{2} \mathrm{O}_{3}$ \\
\hline
\end{tabular}

Em base seca, é importante ressaltar que o valor do resultado é mais preciso para ser utilizado cientificamente. Segundo a NBR 13525 [15], para que o couro seja considerado bem curtido, o teor de óxido de cromo do mesmo precisa ser no mínimo 3,5\% em base seca.

$\mathrm{Na}$ etapa de ribeira, são removidas as partes da pele que não irão constituir o couro e é realizada a preparação para o curtimento, através da preparação das fibras colagênicas. Nesta etapa, se tem os processos de pré-remolho, remolho, depilação e caleiro, pré-descarne e descarne, divisão, desencalagem, purga e píquel. No pré-remolho e no remolho, ocorre a reidratação, além da eliminação de impurezas e o desengraxe. No processo de depilação, a epiderme e os pelos são degradados através de agentes químicos. Em seguida, na operação do caleiro, ocorre a abertura das fibras pelo hidróxido de cálcio. $\mathrm{O}$ cálcio encontrou-se presente neste resíduo, e é proveniente do processo de caleiro, em que é adicionado como hidróxido de cálcio [16]. A matéria volátil representa o teor de água no resíduo, pois a água representa a maior proporção na composição da pele em qualquer etapa no decorrer do processo produtivo [17]. Os valores obtidos para o cromo estão dentro dos limites especificados pela norma e estão de acordo com os valores descritos na literatura. Longhi [16] constatou que o teor de óxido de cromo foi de $4,47 \%$ e o teor de matéria volátil foi de $10,89 \%$. Estes teores de cromo acarretam em mudanças morfológicas na estrutura 
da pele. Conforme Oliveira [18], o couro curtido ao cromo apresenta-se como um material fibroso, evidenciando que o processo de curtimento altera a morfologia da proteína, proporcionando maior estabilidade aos materiais após o curtimento. Isto fica evidenciado através das micrografias, com uma ampliação de 35 vezes, foi possível determinar que o tamanho das partículas do resíduo ficou em torno de $24,2 \mu \mathrm{m}$ a 457,4 $\mu \mathrm{m}$ (Figura 2a). Com uma ampliação de 3000 vezes, pode-se observar que o resíduo do couro é um material fibroso (Figura $2 b$ ).

O espectro de FTIR do resíduo de couro wet-blue (Figura 2a) apresentou banda próximo a $3300 \mathrm{~cm}^{-1}$, a qual pode ser atribuída ao grupo O-H, que segundo Ruiz [19] e Castro [20] correspondem ao estiramento simétrico de grupos presentes no colágeno do resíduo de couro. Na sequência a região entre 1700 e $1620 \mathrm{~cm}^{-1}$ está relacionada ao estiramento da ligação entre carbono e oxigênio $(\mathrm{C}=\mathrm{O})$ de amidas, ligação química presente no resíduo de couro devido aos grupos peptídicos, conforme Santos [21] e Hoque et al. [22].
De acordo com Garcia [23], a banda em 1240,60 $\mathrm{cm}^{-1}$, consiste no estiramento $\mathrm{C}-\mathrm{N}$ e $\mathrm{N}-\mathrm{H}$, devido às interações intermoleculares do colágeno presento no couro. Para finalizar, a banda encontrada em 560,68 $\mathrm{cm}^{-1}$ correspondente ao cromo, como foi observado por Leite [22], no qual verificou que a banda entre 632 e $560 \mathrm{~cm}^{-1}$ atribuía-se ao óxido de cromo. A partir destas análises, também, comprovou-se a formação do material. $O$ espectro do poliuretano puro em comparação com os microcompósitos tem praticamente todas as bandas mais intensas, principalmente as bandas de $\mathrm{CH}_{3}\left(2950 \mathrm{~cm}^{-1}\right)$, $\mathrm{CH}\left(2850\right.$ e $\left.1470 \mathrm{~cm}^{-1}\right), \mathrm{C}=\mathrm{O}\left(1710 \mathrm{~cm}^{-1}\right), \mathrm{NH}(3400 \mathrm{e}$ $\left.1640 \mathrm{~cm}^{-1}\right)$ e $\mathrm{CN}\left(1160 \mathrm{~cm}^{-1}\right)$ [24]. Ou seja, na análise do PU puro, a transmitância em todas estas bandas foi mais intensa. Isso é devido à ausência de carga, pois a intensidade da banda depende da concentração dos componentes da amostra. Ainda, constatou-se a ausência da banda de NCO $\left(2270 \mathrm{~cm}^{-1}\right)$, indicando que a reação foi completa, tanto para o poliuretano puro, como para os microcompósitos. Nota-se, ainda que a banda relativa ao cromo $\left(560,68 \mathrm{~cm}^{-1}\right)$, desapareceu

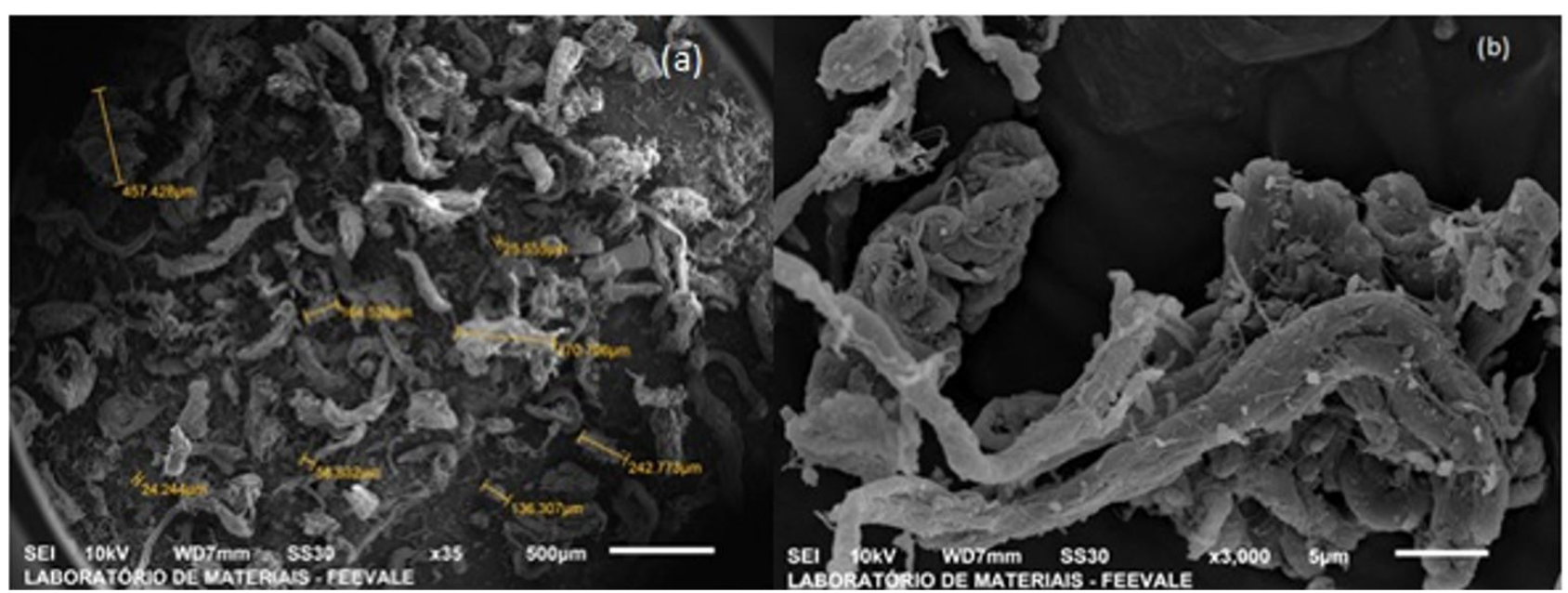

Figura 2. MEV do resíduo wet blue, com uma ampliação de 35 vezes (a) e 3000 vezes (b)

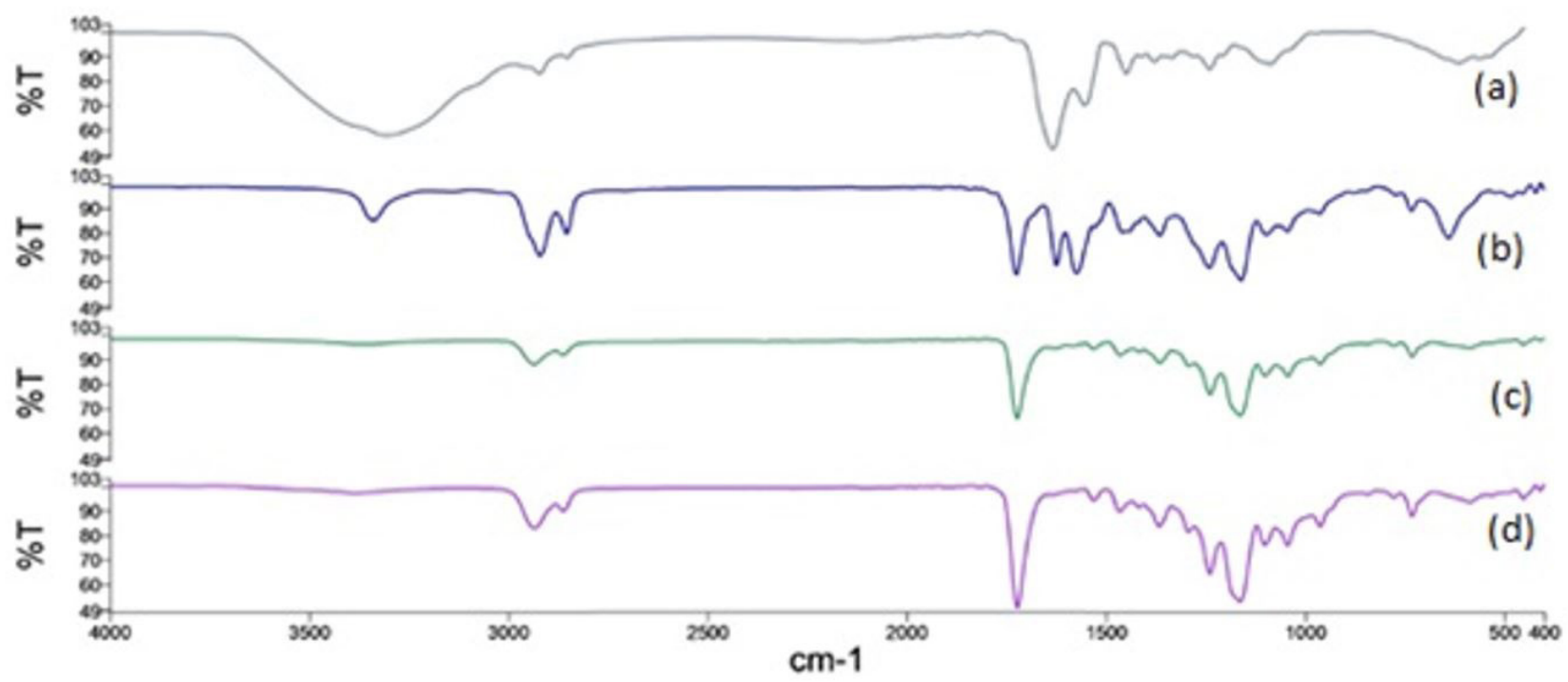

Figura 3. Análises de FTIR do Resíduo wet blue (a), PU puro (b), microcompósito com 5,0\% de resíduo (c) e com 2,0\% (d). 
nos espectros dos microcompósitos, demonstrando que o resíduo foi encapsulado na matriz polimérica. Os espectros estão demonstrados na Figura 3b,3c,3d.

As análises de MEV são apresentadas somente com o poliuretano puro e com o microcompósito com 2,0\% de wet blue para uma melhor visualização. A primeira micrografia, apresentada na Figura 4a, refere-se ao poliuretano puro. Com um aumento de 3000 vezes, pode-se afirmar que sua superfície é bastante lisa e não há interferentes e contaminação, como partículas de outras substâncias. Já na Figura $4 \mathrm{~b}$, tem-se a micrografia do compósito com $2,0 \%$ de resíduo. Nesta imagem é possível observar as partículas do couro e, principalmente, que a polimerização ocorreu de forma que permitisse a interação da carga com o polímero, devido a formação de polímero sobre cada uma das partículas. Este é um bom resultado, sendo que, conforme a literatura, a interação das cargas com a matriz faz com que haja modificação das propriedades do polímero [25]. Dessa forma, pode-se dizer que o método de polimerização in situ mostrou-se eficaz devido à dispersão das partículas no poliuretano, assim como Soares et al. [26], que também citaram as modificações químicas de um compósito de poliuretano com sílica de cinzas de casca de arroz com este tipo de polimerização. Chen et al. [27] realizaram testes em compósitos de PCL com fibra de vidro com base de fosfato e concluíram que por meio da polimerização in situ, as propriedades mecânicas melhoraram significativamente, além da obtenção da distribuição uniforme das cargas.

Nas análises de DRX, foram obtidos os seguintes gráficos, representados na Figura 5, do PU puro (a), microcompósito com $0,5 \%$ de resíduo (b) e com $5,0 \%$ (c). Observou-se o aumento da cristalinidade proporcional ao aumento da quantidade de wet blue adicionado. Conforme Denchev [28], um maior grau de cristalinidade em um material faz com que ele tenha maior resistência mecânica, maior densidade, maior resistência térmica, maior rigidez e maior resistência a solventes. Então, mesmo sem ter sido realizadas mais análises para a determinação dessas propriedades citadas, pode-se esperar que elas aumentaram nos microcompósitos conforme o aumento da concentração de carga. Em um estudo sobre tratamentos com alcáli e ultrassom em fibras de sisal para incorporação em polipropileno, foi concluído, por meio de análise DRX, que a cristalinidade aumentou com o aumento na concentração de álcali [29]. Isso reforça os resultados obtidos, em que houve o aumento da cristalinidade dos microcompósitos com o aumento da concentração de resíduo de couro wet blue devido à presença de cromo. Khederlou et al. [30], caracterizaram, por DRX, a dispersão de nanopartículas de argila em compósitos híbridos de polietileno de baixa densidade (PEBD) e polietileno linear de baixa densidade (PELBD). Os autores concluíram que o aumento da argila nos compósitos resultou em melhoria significativa na interação entre as cadeias dos polímeros. Consequentemente, esta interação modifica as propriedades do material conforme a concentração da carga, assim como ocorreu com a adição do resíduo wet blue no poliuretano e foi apresentado com as análises realizadas.

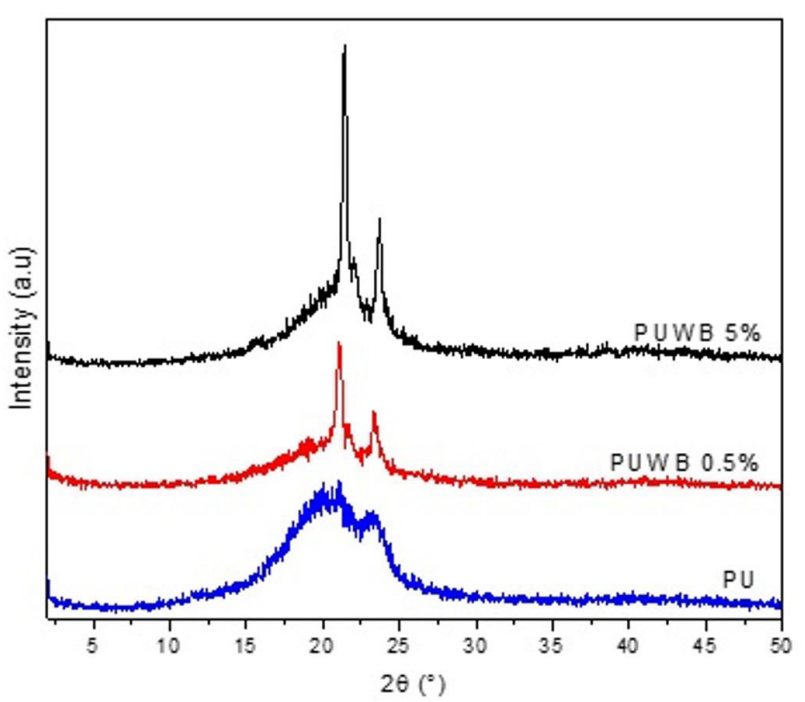

Figura 5. Análises de DRX do PU puro e dos microcompósitos com 0,5 e $5,0 \%$ de resíduo wet blue.

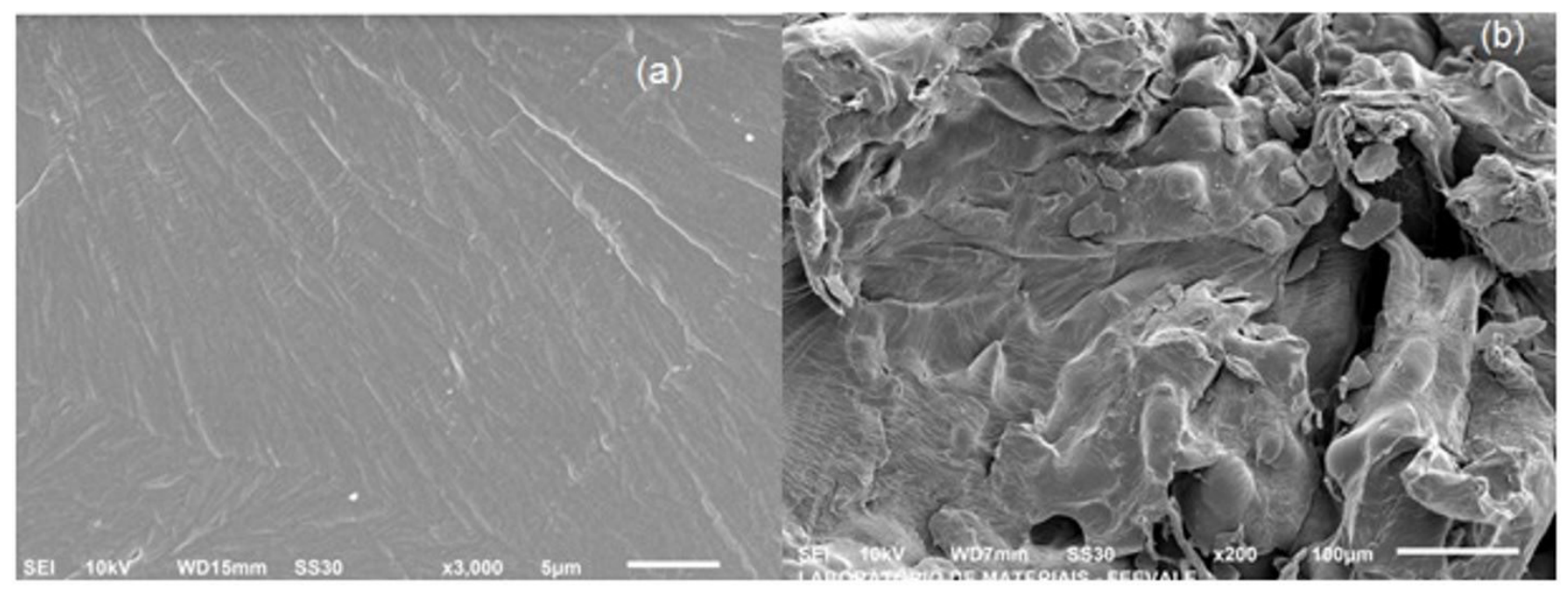

Figura 4. Micrografia do PU puro (a) e do microcompósito com 2,0\% de resíduo. 
Por meio da análise de TGA, foi determinada a estabilidade térmica dos microcompósitos. Esta técnica é uma ferramenta importante para a compreensão dos mecanismos de degradação dos polímeros [31]. Os resultados da análise estão demonstrados na Tabela 2. Na Figura 6 são apresentados os gráficos para o PU puro (a), 5,0\% (b), em que a linha contínua vermelha refere-se à curva $\mathrm{TG}$ e a linha tracejada azul refere-se à curva DTG.

Primeiramente, observou-se por meio das curvas TG e DTG que em todas as análises a decomposição principal das amostras ocorreu em uma única etapa. $\mathrm{O}$ poliuretano puro começou a degradar na temperatura de $324,49{ }^{\circ} \mathrm{C}$. Por meio da curva DTG, determinou-se como $373,00{ }^{\circ} \mathrm{C}$ a temperatura em que a taxa de variação de massa é máxima,

Tabela 2. Temperatura de degradação térmica e \% do aumento em relação ao PU puro

\begin{tabular}{ccc}
\hline MATERIAL & $\begin{array}{c}\text { T }\left({ }^{\circ} \text { C) INÍCIO }\right. \\
\text { DEGRADAÇÃO }\end{array}$ & $\begin{array}{c}\text { AUMENTO EM } \\
\text { RELAÇÃO AO PU } \\
\text { PURO (\%) }\end{array}$ \\
\hline Poliuretano puro & 325 & - \\
Microcompósito com & 328 & 1 \\
$0,5 \%$ de wet blue & & 6 \\
$\begin{array}{c}\text { Microcompósito com } \\
\text { 2,0\% de wet blue }\end{array}$ & 345 & 12 \\
$\begin{array}{c}\text { Microcompósito com } \\
5,0 \% \text { de } \text { wet blue }\end{array}$ & 362 & 12 \\
\hline
\end{tabular}

conforme a Figura 5a. Nos micrompósitos, a resistência térmica aumentou com o aumento da quantidade de resíduo adicionado em relação ao polímero puro. No material com 5\% de wet blue, a temperatura em que iniciou-se a degradação foi de $362,26^{\circ} \mathrm{C}$, ou seja, $11,639 \%$ maior que no poliuretano puro. Este aumento de resistência térmica observado ficou de acordo com a literatura. Peng-Gang et al. [32] afirmaram que a dispersão das cargas em compósitos poliméricos tem grande influência na estabilidade térmica que esses materiais apresentam, pois eles estudaram a dispersão de cargas por meio de diferentes técnicas de moldagem por compressão. Em um estudo sobre nanocompósitos de espuma rígida de poliuretano (PUR), Saha et al.[30], afirmaram que com a adição das nanocargas, os compósitos tiveram temperatura de degradação térmica maior, ou seja, as cargas fizeram com que as cadeias de PUR se mantivessem intactas a temperaturas mais elevadas do que no polímero puro. O comportamento térmico das amostras foi avaliado através do DSC. Os resultados estão demonstrados na Tabela 3.

Os resultados representados na Tabela 3 mostram que as amostras apresentaram um pico endotérmico correspondente à fusão e um pico exotérmico correspondente à cristalização. $\mathrm{O}$ resíduo de couro wet-blue apresentou um pico endotérmico único próximo a $62{ }^{\circ} \mathrm{C}$ decorrente da desnaturação do colágeno do couro, segundo Santos [21], no qual ressaltou que a temperatura de desnaturação do couro está diretamente relacionada a rede de reticulação formada
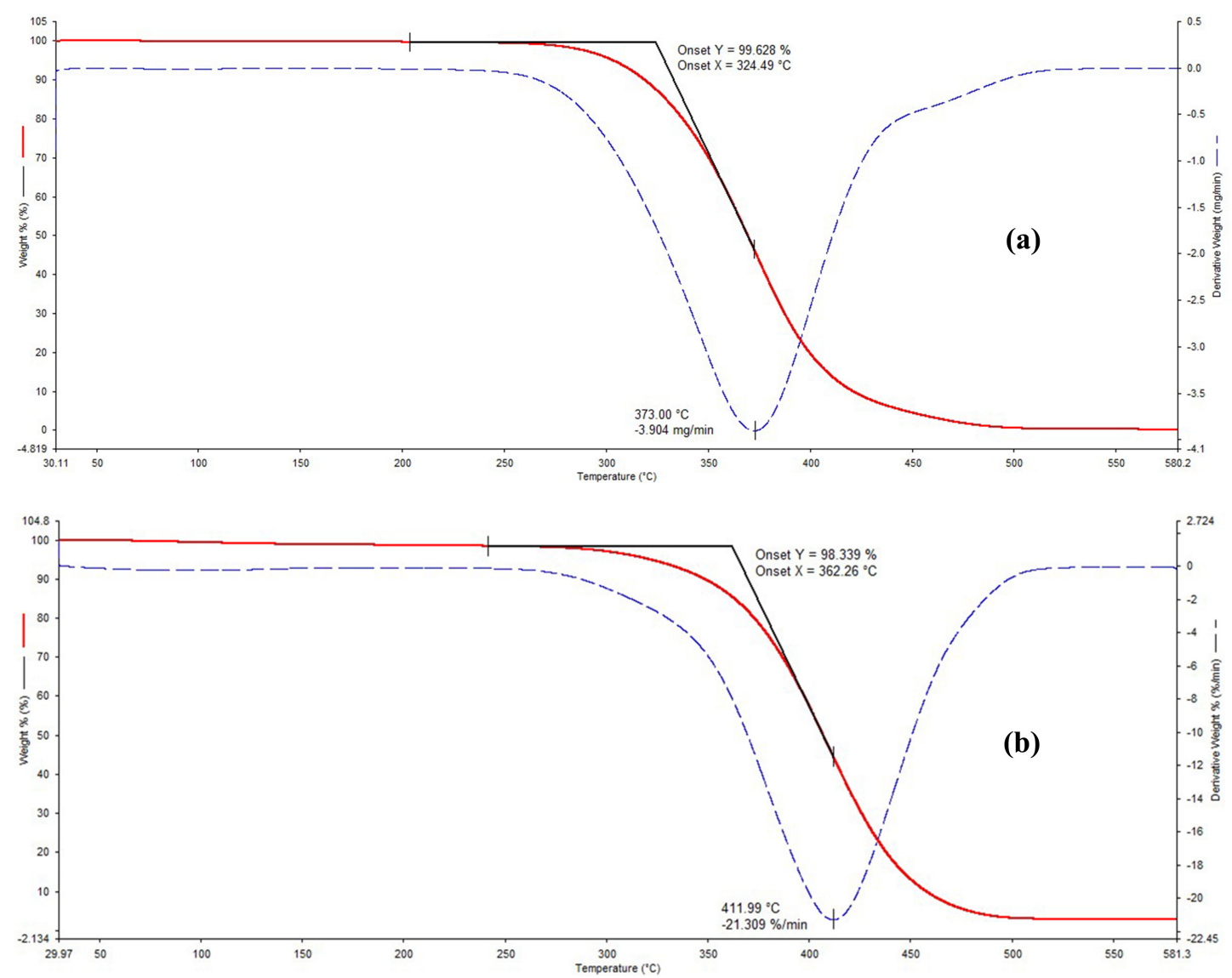

Figura 6 [[Q3: Q3]]. TGA do PU puro (a) e do Microcompósito com 5\% (b) de resíduo wet blue. 
Tabela 3. Parâmetros obtidos a partir da curva de DSC para a amostra de resíduo de couro wet blue, pu puro e microcompósitos com 2,0 e $5,0 \%$ de resíduo wet blue

\begin{tabular}{ccccc}
\hline \multirow{2}{*}{ AMOSTRA } & $\mathbf{T c}\left({ }^{\circ} \mathbf{C}\right)^{1}$ & $\begin{array}{c}\Delta \mathbf{H c} \\
(\mathbf{J} / \mathbf{g})^{2}\end{array}$ & $\begin{array}{c}\text { Tm } \\
\left({ }^{\circ} \mathbf{C}\right)^{3}\end{array}$ & $\begin{array}{c}\Delta \mathbf{H m} \\
(\mathbf{J} / \mathbf{g})^{4}\end{array}$ \\
\cline { 2 - 5 } & - & - & 62,47 & 466,82 \\
\hline $\begin{array}{c}\text { Resíduo de couro } \\
\text { wet-blue }\end{array}$ & $-6,58$ & $-24,18$ & 33.44 & 26,73 \\
$\begin{array}{c}\text { PU puro } \\
\text { Microcompósito com }\end{array}$ & $-1,86$ & $-38,94$ & 37,54 & 38,20 \\
$\begin{array}{c}\text { 2,0\% de wet blue } \\
\text { Microcompósito com } \\
\text { 5,0\% de wet blue }\end{array}$ & 2,55 & $-40,62$ & 40,74 & 26,02 \\
\hline
\end{tabular}

${ }^{1}$ Temperatura de cristalização; ${ }^{2}$ Entalpia de cristalização; ${ }^{3}$ Temperatura de fusão; ${ }^{4}$ Entalpias de fusão.

pelo cromo na estrutura do colágeno e também a fatores de natureza biológica.

Em relação aos materiais sintetizados, observou-se um aumento na temperatura de cristalização, de $-6,58^{\circ} \mathrm{C}(\mathrm{pu}$ puro) à $2,55{ }^{\circ} \mathrm{C}$ (Microcompósito com $5,0 \%$ de wet blue), indicando que o resíduo atuou como agente nucleante [33], efeito também observado por Dias [34], que preparou compósitos de poliuretano com talco - Ni. De forma geral, as amostras apresentaram um deslocamento dos picos das curvas endotérmicas, relativos à temperatura de fusão, quando comparadas ao material sem adição de resíduo, variando de $33,44^{\circ} \mathrm{C}$ (pu puro) à $40,74{ }^{\circ} \mathrm{C}$ (Microcompósito com $5,0 \%$ de wet blue). Este comportamento foi observado, também, por Pluta et. al. [35], que verificou que a temperatura de fusão tem direta correlação com o percentual de carga utilizada na formulação. Logo, pôde-se inferir que a inserção da carga de couro wet-blue nas proporções estudadas proporcionou uma estabilidade térmica, através de um aumento da cristalinidade dos materiais desenvolvidos, corroborando com os resultados encontrados nas análises de TGA.
Um aspecto interessante, é a proposição de que a formação de compostos com características variadas, atenderia às necessidades ambientais das empresas por meio de processos de reciclagem interna. Também, que a utilização deste resíduo como reforço em materiais compósitos, apresenta um grande potencial para melhorar o desempenho de polímeros além de apresentar baixo custo, abundância [36].

\section{Conclusões}

Os resultados obtidos na realização desta pesquisa foram satisfatórios devido às propriedades agregadas ao poliuretano e às análises escolhidas, que caracterizaram adequadamente os materiais. Foi obtido um aumento da estabilidade térmica e do grau de cristalinidade dos microcompósitos quando comparados ao PU puro. Principalmente quando a concentração da carga foi maior, maiores foram as modificações das propriedades, estando de acordo com o que há na literatura. O microcompósito com a concentração de 5,0\% em massa de resíduo de couro wet blue foi o material que apresentou os melhores resultados. A sua resistência térmica ficou maior do que no PU puro e nos demais microcompósitos. A temperatura em que o material começou a degradar termicamente foi $11,639 \%$ maior que a temperatura do polímero puro, chegando a $362,26{ }^{\circ} \mathrm{C}$. Sua cristalinidade, consequentemente, também foi a maior entre os materiais sintetizados. Esta melhora nas propriedades térmicas gera a oportunidade para esses microcompósitos serem utilizados em diferentes aplicações, principalmente, no setor coureiro calçadista, gerando economia de recursos. Além de reutilizar um resíduo classe I que é gerado em grandes quantidades e que pode causar sérios danos ambientais dependendo das condições em que é depositado ou reciclado.

\section{Referências}

1 Associação Brasileira de Normas Técnicas. ABNT NBR 10.004: Resíduos Sólidos - Classificação. Rio de Janeiro, RJ: ABNT; 2004.

2 Centro das Indústrias de Curtumes do Brasil. O couro e o curtume brasileiro. Brasília, DF: CICB; 2016 [acesso em jul. 2018]. Disponível em: http://www.cicb.org.br

3 Fundação Estadual de Proteção Ambiental - FEPAM. Plano Estadual de Resíduos Sólidos do Rio Grande do Sul. Porto Alegre: FEPAM; 2014.

4 Hu J, Xiao Z, Zhou R, Deng W, Wang M, Ma S. Ecological utilization of leather tannery waste with circular economy model. Journal of Cleaner Production. 2011;19:221-228.

5 Gil RR, Girón RP, Lozano MS, Ruiz B, Fuente E. Pyrolysis of Biocollagenic wastes of vegetable tanning, optimization and kinetic study. Journal of Analytical and Applied Pyrolysis. 2012;97:129-136.

6 El-Sabbagh SH, Mohamed OA. Recycling of chrome-tanned leather waste in Acrylonitrile Butadiene rubber. Journal of Applied Polymer Science. 2011;121:979-988.

7 Bañón E, Marcilla A, García AN, Martínez P, León M. Kinetic model of the thermal pyrolysis of chrome tanned leather treated with Naoh under different conditions using thermogravimetric analysis. Waste Management. 2016;48:285-299. 
8 Gutterres, M. Alternativas para destinação do resíduo do rebaixamento de couro Wet Blue. Revista do Couro. 1996;113(22):49-54.

9 Guo X, Hou WH, Ding W, Fan Y, Yan Q, Chen Y. Synthesis of novel inorganic-organic hybrid nanocomposites: Intercalation behaviour of titanium(Iv) (Triethanolaminato)-Isopropoxide in a series of layered protonic metal oxides. Inorganic Chemistry Communications. 2004;7:946-948.

10 Essabir H, Boujmal R, Bensalah MO, Rodrigue D, Bouhfid R, Qaiss A. Mechanical and thermal properties of hybrid composites: Oil-palm fiber/clay reinforced high density Polyethylene. Mechanics of Materials. 2016;98:36-43.

11 Riehl A. Caracterização de resíduos de couro provenientes de um aterro industrial [dissertação]. São Leopoldo: Universidade do Vale dos Sinos; 2015.

12 Qu R, Gao J, Tang B, Ma Q, Qu B, Sun C. Preparation and Property of Polyurethane/Nanosilver Complex Fibers. Applied Surface Science. 2014;294:81-88.

13. Silva MA, Tavares MIB, Nascimento SAM, Rodrigues E, Caracterização De nanocompósitos de poliuretano/ montmorilonita organofílica por RMN de baixo campo. Polímeros Ciência e Tecnologia. 2012;22(5):481-485.

14 Associação Brasileira de Normas Técnicas. ABNT NBR 11054: Couro - Determinação de óxido crômico total. Rio de Janeiro: ABNT; 1999.

15 Associação Brasileira de Normas Técnicas. ABNT NBR 13525: Determinação de Óxido Crômico Totalizado de Resíduos Sólidos. Rio de Janeiro: ABNT; 2016.

16 Longhi EM. Obtenção de nutrientes para plantas oriundos de resíduo do couro [dissertação]. Novo Hamburgo, RS: Universidade Feevale; 2018.

17 Amaral LA. Alternativas Para o tratamento de resíduos de couro curtido ao cromo - Hidrólise enzimática e ação bacteriana [dissertação]. Porto Alegre, RS: Universidade Federal do Rio Grande do Sul; 2008.

18 Oliveira R. Contaminação do solo por alguns resíduos de curtume [tese]. Viçosa, MG: Universidade Federal De Viçosa; 2008.

19 Ruiz MR. Caracterização do compósito obtido a partir de borracha natural com negro de fumo e resíduos de couro aplicado como pisos e revestimentos [tese]. Presidente Prudente, SP: Universidade Estadual Paulista Júlio de Mesquita Filho; 2014.

20 De Castro IA. Resíduo de couro Wet-Blue após a extração do cromo: uso como fertilizante nitrogenado em plantação de eucalipto [dissertação]. Lavras, MG: Universidade Federal de Lavras; 2011.

21 Dos Santos RJ. Obtenção de compósitos de borracha natural com resíduo industrial de couro reticulados com diferentes peróxidos [tese]. Presidente Prudente, SP: UNESP; 2014.

22 Hoque MS, Benjakul S, Prodpran T. Effects of partial hydrolysis and plasticizer content on the properties of film from Cuttlefish (Sepia Pharaonis) skin gelatina. Food Hydrocolloids. 2011;25(1):82-90.

23 Garcia NG. Descontaminação do resíduo industrial de couro, uma proposta para o desenvolvimento sustentável nos curtumes [dissertação]. Presidente Prudente, SP: Universidade Estadual Paulista Júlio De Mesquita Filho; 2015.

24 Silva V, Santos L, Subda S, Ligabue R, Seferin M, Carone CLP, et al. Synthesis and characterization of Polyurethane/Titanium dioxide nanocomposites obtained by in situ polymerization. Polymer Bulletin. 2013;70:1819-1833.

25 De Lima V. Estudo De catalisadores organometálicos na síntese de poliuretanos [dissertação]. Porto Alegre, RS: PGETEMA-PUCRS; 2007.

26 Soares R, Monteiro W, Andréa C, Lima V, Carone CLP, Dullius J, Seferin M, Einloft S, Ligabue R. Compósitos Poliméricos obtidos pela incorporação de sílica proveniente de cinzas da casca de arroz. In: Anais do $13^{\circ}$ Simpósio Latinoamericano de Polímeros. 2012 Setembro 23-26; Bogotá, Colombia. Bogotá: SLAP; 2012.

27 Chen M, Parsons AJ, Felfel RM, Rudd CD, Irvine DJ, Ahmed I. In-situ Polymerisation of fully bioresorbable polycaprolactone/phosphate glass fibre composites in vitro degradation and mechanical properties. Journal of the Mechanical Behavior of Biomedical Materials, 2016;59:78-89.

28 Dencheva N, Stribeck A, Denchev Z. Nanostructure development in multicomponent Polymersystems characterized by synchrotron X-ray scattering. European Polymer Journal. 2016;81:447-469.

29 Krishnaiah P, Ratnam CT, Manickam S. Enhancements in crystallinity, thermal stability, tensile modulus and strength of sisal fibres and their pp composites induced by the synergistic effects of alkali and high intensity ultrasound (HIU) treatments. Ultrasonics Sonochemistry. 2017;34:729-742. 
30 Khederlou K, Bagheri R, Shojaei A. A mathematical method for XRD pattern interpretation in clay containing nano composites. Applied Surface Science. 2014;318:90-94.

31 Chattopadhyay DK, Webster DC. Thermal stability and flame retardancy of Polyurethanes. Progress in Polymer Science. 2009;34:1068-1133.

32 Peng-Gang R, Si-Yu H, Fang R, Zeng-Ping Z, Zhen-Feng S, Ling X. The influence of compression molding techniques on thermal conductivity of Uhmwpe/Bn And Uhmwpe/(Bn + Mwent) hybrid composites with segregated structure. Composites. Part A, Applied Science and Manufacturing. 2016;90:13-21.

33 Saha MC, Kabir ME, Jeelani S. Enhancement in thermal and mechanical properties of Polyurethane foam infused with nanoparticles. Materials Science and Engineering A. 2008;479:213-222.

34 Dias G, Prado M, Carone CLP, Ligabue R, Dumas A, Le Roux C, et al. Comparing different synthetic talc as fillers for polyurethane nanocomposites. Macromolecular Symposia. 2016;367:136-142.

35 Pluta M, Murariu M, Alexandre M, Galeski A, Dubois P. Polylactide Compositions. the influence of ageing on the structure, thermal and viscoelastic properties of PLA/calcium sulfate composites. Polymer Degradation \& Stability. 2008;93(5):925-931.

36 Borsoi C, Scienza LC, Zattera AJ, Angrizani, C. Obtenção e caracterização de compósitos utilizando poliestireno como matriz e resíduos de fibras de algodão da indústria têxtil como reforço. Polímeros: Ciência e Tecnologia. 2011;21(4):271279.

Recebido em: 29 Mar. 2019

Aceito em: 05 Abr. 2020 